\title{
High Temperature Superconductivity: A Prospective Remedy for Energy Crisis in Future
}

\author{
Chhabi L. Gnawali, Binod Pandey a \\ Dept. of Applied Sciences \& Chemical Engineering, Pulchowk Campus, Institute of Engineering, Tribhuvan University, Nepal \\ Corresponding Author: ${ }^{a}$ pandeybinodeioe.edu.np
}

Received: 2020-08-06

Revised: 2021-02-05

Accepted: 2021-02-19

\section{Abstract:}

This paper summarizes the superconducting phenomenon followed by application of superconductivity devices on energy sector. High temperature superconductivity are very innovative, promising and eco-friendly alternative to mitigate the energy crisis by saving energy.

Keywords: Superconductivity, Eco-friendly, Energy

\section{Introduction}

Twentieth century, a prolific span of scientific achievement was also marked by the dawn of enthralling quantum phenomenon on macroscopic scale 'a superconductivity' $[3,16]$. Its origin can be traced backed to the accidental discovery of technique to liquefy chorine by Michael Faraday to breakthrough in liquefy Helium by Onnes in 1908. As Onnes has access to the world lowest temperature in his laboratory he was on the place to settle an outstanding scientific problem, the resistance of a metal as it was cooled to absolute zero. Onnes choose a mercury and found that when the sample was slowly cooled below the boiling point of liquid Helium (4.2K). The resistance of the sample disappeared at $4.15 \mathrm{~K}$ suddenly and if the temperature rises the sample revert to normal state. This was an event of discovery of superconductivity in 1911.

Experimentation on different metal like $\mathrm{Pb}, \mathrm{Sn}$, subsequently established that the resistivity is indeed zero below the critical temperature $\left(\mathrm{T}_{\mathrm{c}}\right)$ which however depends on the superconducting elements. Another very important aspect of superconductor, unveiled by W Meissner and R Ochsenfeld in 1933 is the expulsion of magnetic flux when superconductor is cooled below the critical temperature in a magnetic field. This perfect diamagnetic property of superconductor is the most fundamental macroscopic property of superconductor which also leads to differentiate superconducting property with perfect conductor. For perfect conductor resistivity is zero, i.e., from Ohms law $(E=\rho J)$ no electric field can exist inside the perfect conductor. Using Maxwell's equations

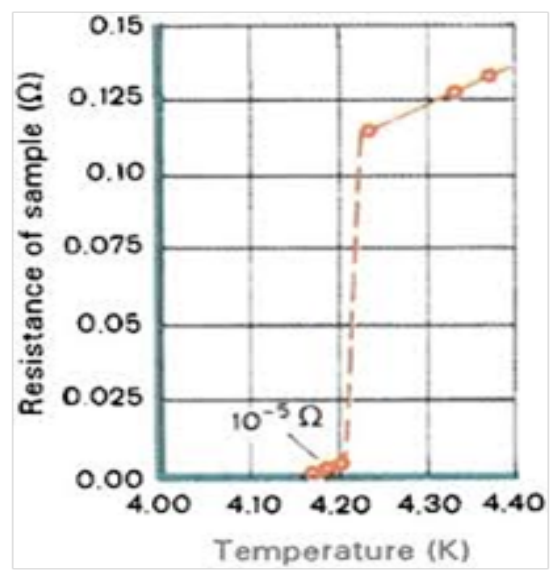

Figure 1: Discovery of superconductivity by Kamerlingh Onnes [11]

$$
\nabla \times \vec{E}=-\frac{\partial \vec{B}}{\partial t}
$$

We get, $\mathrm{B}=$ constant

This result predict that flux through perfect conductor remain unaltered as it is cooled in the magnetic field until its resistance become zero. However Meissner effect associated with superconductor contradicts this result $[3,11]$. 


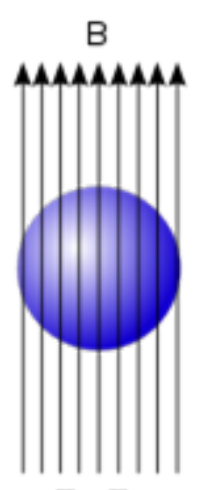

$T>T_{C}$

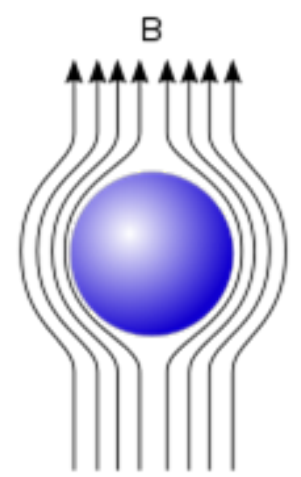

$T<T_{C}$
Figure 2: Meissner effect [23]

\section{Types of superconductor}

Superconductor can be classified into two categories depending upon their response to external magnetic field.

Pure specimen of many material like $\mathrm{Pb}, \mathrm{Hg}, \mathrm{Sn}, \mathrm{Al}$ and other exhibit complete Meissner effect i.e., total expulsion of magnetic field below a certain critical magnetic field $\mathrm{Bc}$ at a given temperature and at $\mathrm{B}=\mathrm{Bc}$, loses superconductivity an revert to normal conductor form a type I superconductor. These are conventional superconductor whose critical temperature is below $25 \mathrm{~K}$. The critical magnetic field depends upon the temperature and appears to follow the relation expressed as

$$
B_{c}(T)=B_{c}(0)\left[1-\left(\frac{T}{T_{c}}\right)^{2}\right]
$$

Where $B_{c}(T)$ represents the critical magnetic field at temperature $T, B_{c}(0)$ represents the critical magnetic field at zero kelvin. Tc is transition temperature.

Superconducting alloy like lead-indium, niobium-titanium, niobium-tin as well as high temperature superconductor like $\mathrm{HgBa}_{2} \mathrm{Ca}_{2} \mathrm{Cu}_{3} \mathrm{O}_{8}$, $\mathrm{Bi}_{2} \mathrm{Sr}_{2} \mathrm{CaCu}_{2} \mathrm{O}_{8+\delta}$ etc. form a hard or a type II superconductor. They are characterized by two critical field $\mathrm{B}_{\mathrm{c} 1}$ and $\mathrm{B}_{\mathrm{c} 2}$. Below $\mathrm{Bc} 1$ these substance are in Meissner phase with complete field expulsion while in the range $\mathrm{B}_{\mathrm{c} 1}<\mathrm{B}<\mathrm{B}_{\mathrm{c} 2}$, type II superconductor enter the mixed phase in which magnetic field can penetrate the bulk material. However the superconductivity is still maintained at this phase. The superconductivity vanishes above the $B_{c 2}$ which is much higher critical field enabling type II for its technical utilities. The magnetization curve for Type I and type II superconductor are depicted in Figure 3 [3, 11, 13].
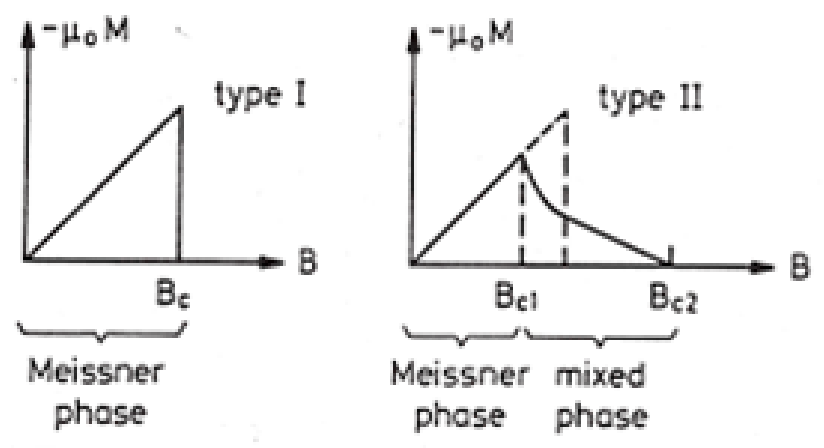

Figure 3: Magnetization versus applied magnetic field for Type I and Type II superconductor [13]

\section{London Equation}

The first successful explanation of the Meissner Ochsenfeld effect was achieved in 1935 by Heinz and Fritz London. They assumed that the supercurrent is carried by a fraction of the conduction electron called superconducting electron. These super current is distributed in the surface layer which lead to exponential attenuation of external magnetic field inside the superconductor and described by the equation

$$
B(x)=B_{e x t} \exp \left(\frac{-x}{\lambda}\right)
$$

Where $x$ represent the distance from the surface to the specimen and Bext is the field at external surface. The parameter $\lambda$ gives the depth of penetration where magnetic field decrease to $1 / e$ of the surface known as London penetration depth. This London penetration depth depend strongly on the temperature and follows the relation

$$
\lambda(T)=\frac{\lambda(0)}{\sqrt{1-\left(\frac{T}{T_{c}}\right)^{4}}}
$$

$\lambda(T)$ is penetration depth at temperature $\mathrm{T} K$.

$\lambda(0)$ is penetration depth at temperature $0 \mathrm{~K}[2,13]$ 


\section{Theory of Superconductivity: BCS Theory}

Leon Cooper, an American physicist using the field theory show that an arbitrary small attraction between electrons can form a bound system of two electron with an energy less than twice fermi energy, $\mathrm{E}_{\mathrm{pair}}<2 \mathrm{E}_{\mathrm{F}}$.

This lead to realize Bardeen, Cooper and Schrieffer that the electron inside the fermi sphere could also able to group into pair follow by reduction in their energy. However attractive interaction to pair up is attributed to electron-lattice-electron or electron-phonon-electron interaction. According to BCS theory as one negatively charged electrons passes by positively charged ions in the lattice of superconductor the lattice distorts. This lattice deformation give rise to excess charge that attracts the second electron of equal and opposite momentum and spin. These electron form a cooper pair which extend from 100-1000 lattice constant apart. The cooper pairs occupy a single quantum state and behave as a boson with spin 0.The highest possible phonon energy is in the range of $0.01-0.02 \mathrm{ev}$ which ultimately restrict the fraction of electron to be paired via phonon exchange.

The formation of Cooper pairs leads to energy gap in superconductor which depends on temperature. Coopers pairs exists only in the BCS ground state and an excitation is equivalent to breaking them up into single electrons.

BCS theory of superconductivity employ quantum mechanics which endow wavelike behavior to electron and described by a wave function that pervade throughout the solid and overlap with other electron wave function. Consequently the whole network of electron behave like one wave function so that their collective motion is coherent $[2,3,6,10,13]$.

\section{High Temperature Superconductivity}

The BCS theory which explained everything that has been so far discovered doesn't underpin the discovery of high temperature superconductivity which is in line with the record transition temperature stuck at $23 \mathrm{~K}$ since 1973. However the $T_{c}$ of $30 \mathrm{~K}$ reported for lanthanum barium copper oxide by $\mathrm{K}$ Muller and $\mathrm{J}$ Bednorz in 1986 was a silver lining for the high temperature superconductivity. This was followed by a key breakthrough in 1987 by Chu and his colleague, synthesis of Yttrium compound with a superconducting transition temperature of $93 \mathrm{~K}$.

The discovery of first high temperature superconductor in 1986 encourage many researcher in quest of new material having a high critical temperature. As a result many new material were discovered including modification of earlier material. The highest $T_{c}$ recorded till now is $139 \mathrm{~K}$ which has been obtained for a mercury thallium copper oxide at ambient pressure. These are remarkable since it surpasses the boiling point of liquid nitrogen $(77 \mathrm{~K})$ and even rekindle the interest in superconductivity from the perspective of technological application [2, 3]. While for many years cuprates remains as a single candidates of HTS. Figure 4 shows the time evolution of Transition temperature $\left(\mathrm{T}_{\mathrm{c}}\right)$ of the conventional and high temperature superconductor.

By the beginning of 21st century there are some key achievement in superconductivity. In 2001 Japanese scientist Akimitsu and his team discovered a superconductivity on $\mathrm{MgB}_{2}$ which has a $\mathrm{T}_{\mathrm{c}}$ of $40 \mathrm{~K}$. It was an exciting event to have superconductivity on cheap and simple compound [4].

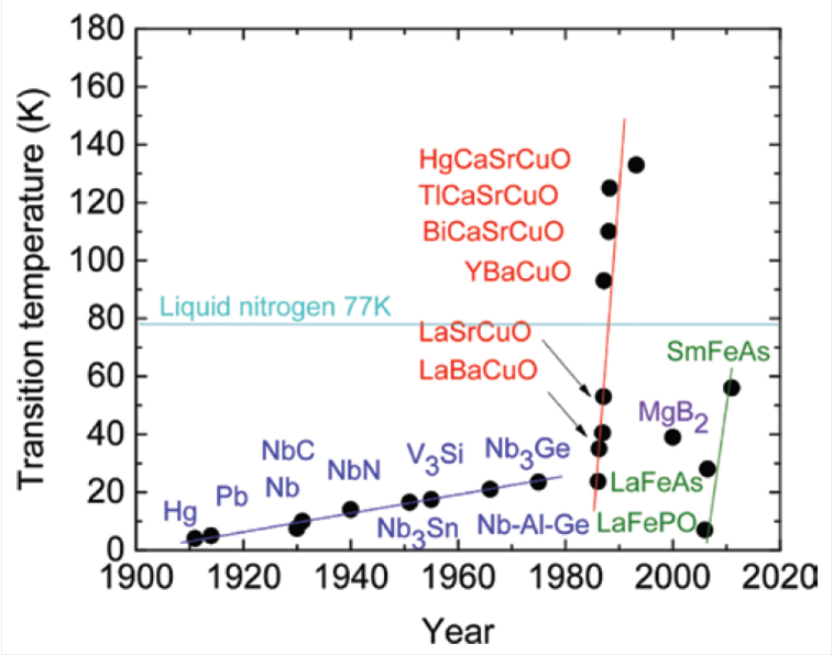

Figure 4: Time evolution of Conventional and high temperature superconductor [4]

In late 2007, Hideo Hosono and coworkers announced the discovery of high temperature superconductivity in pnictide. The maximum $\mathrm{T}_{\mathrm{c}}$ is $56 \mathrm{~K}$ on the pnictide compound by 2008. This new superconductor is without copper atom and consists of iron arsenic layer interleaved with the samarium oxygen layers. Like copper oxide superconductor, iron pnictide also have layered structure. Furthermore, both of them are 
magnetic and achieve superconducting properties after optimal chemical doping [4].

More recently in 2019, Drozdev et al. investigated the superconductivity of lanthanum hydrides. They found the record breaking critical temperature Tc of $250 \mathrm{k}$ at the pressure of $170 \mathrm{GPa}$. This is an encouraging steps towards the goal of achieving room temperature superconductor at ambient pressure in the near future [7].

There are large number of theory and model proposed to explain superconductivity such as magnon model, exciton model, bipolaronic model, model of resonant valance bonds, plasmon model to mention some of them. However a consensus regarding the pairing mechanism of carriers has not been reached among researchers $[5$, $16,20,21]$.

Many researcher believes that phonon are responsible for high temperature superconductivity. It is further backed by the explanation of superconducting properties of magnesium diboride of Tc $40 \mathrm{~K}$ by electron phonon mechanism [20, 21].

Some recent theoretical model however postulated antiferromagnetic fluctuation for pairing mechanism. It is evident in the High Temperature superconductor that superconductivity wave function have d-wave symmetry. Electrons in high temperature compounds pairs in $d_{X^{2}-Y^{2}}$ state and the occupancy of this state favor the spin fluctuation mechanism of pairing $[1,6$, $16,21]$.

Valla and team experiment on $\mathrm{Bi}_{2} \mathrm{Sr}_{2} \mathrm{CaCu}_{2} \mathrm{O}_{8+\delta}$ rule out the phononic mechanism of pairing and supports the spin fluctuation as a possible cause of pairing process [22].

Copper oxide high temperature superconductor have a layered structure consisting of $\mathrm{CuO}_{2}$ layer separated by insulating layers. Superconductivity occur in $\mathrm{CuO}_{2}$ layers and the insulating layers acts as a charge reservoir. Some models prefer the interlayer coupling model which consider the tunneling interaction between the layers as responsible mechanism for HTS $[1,6,16]$.

\section{Superconductivity with Energy}

Rapid growth on population, influx of population towards cities as well as transformation of cities to smart cities led to rise on demand of energy. The existing electricity grid are the route of supply of the energy. However restricted current density of conventional cable made up of copper and alumunium entail the installation of new lines to accommodate increasing demand midst global transmission and distribution losses of $8.26 \%$ in 2014 and public opposition for new corridors of transmission lines [8, $14,18]$.

With the advent of HTS, superconducting cable has create a ground to be a promising option to accommodate the increasing demand on electricity grid. Compactness and flexibility are the major features of superconducting cable which allow to conduct 100 times the electrical current or can carry equivalent power at much lower voltage in comparison to the conventional cable of similar dimensions.

Apart from the fact that the superconducting cable have no resistive losses, presence of superconducting shield also eliminate the eddy current loss from the electromagnetic field. Figure 5 shows the comparison of transmission losses in a superconducting and conventional cable for $66 \mathrm{KV}, 3 \mathrm{KA}, 350 \mathrm{MW}$ power, the loss of superconducting cable is about $50 \%$ of the conventional cable.

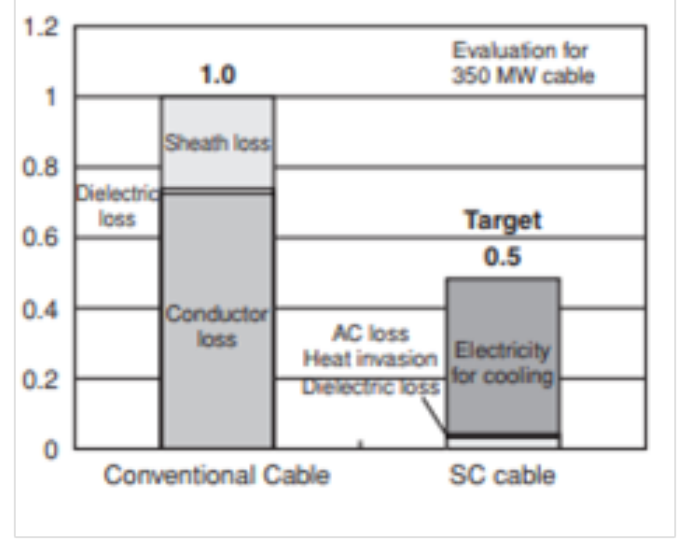

Figure 5: Transmission loss [15]

Besides this, no load losses in HTS are larger in comparison to losses in conventional cable, therefore superconducting cable are appropriate for major cities , bussiness and industrial area where high load are connected for large part of time [4, 9, 12, 15, 19].

Superconducting cable system are much lighter and compact than conventional cable lead to reduction of raw material ,energy and greenhouse gases emission associated with manufacturing process. In practice, superconducting cable are installed underground which 
help to get rid of strong public opposition usually encountered in overhead transmission lines due to concerns over their environmental and visual impact, falling property prices and the health risks posed by electromagnetic fields. In addition, low transmission loss not only provides energy for large number of end user but also help to curb the $\mathrm{Co} 2$ emission since fossil fuel are the primary source for energy generation $[9,15$, 19].

Superconducting magnet are the backbone of International Thermonuclear Experimental Reactor (ITER). Intense magnetic field produced by these magnets are responsible for plasma confinement and stabilization which are paving a way to set up , magnetic confinement fusion as a potential candidate sources of eco-friendly energy [17].

Superconducting magnetic energy storage is one of the energy storing technology which is used to store the spare energy in superconducting coils as a magnetic field. In addition, the magnetic levitating train use a superconducting magnet which unlike conventional trains are highly efficient and avoid energy losses from friction $[3,4,16]$.

\section{Conclusion}

Superconductor though itself not a sources of energy but a vital tools for generation, transmission and storage of the energy. High temperature superconductor are very innovative, promising and eco-friendly alternative to mitigate the energy crisis because of their efficiency and flexibility. Despite its application in the sector of medical imaging and particle accelerator since long, it is still in infancy in the energy sector. However we can anticipate for cost competitive high temperature superconductor or even room temperature superconductor.

\section{Acknowledgment}

Authors are grateful to Prof. Dr. Bhadra Pokharel for his constant guidance and motivation.

\section{References}

[1] S. Bansal S, "High Temperature Superconductivity: A Review," International Journal of Scientific Research \& Development, vol. 3, no. 6, pp. 305-306, 2015.
[2] D. K. Bhattacharya and P. Tandon, Engineering Physics. Oxford University Press, 2015.

[3] S. Blundell, Superconductivity. Oxford University Press, 2015.

[4] A. Bussmann-Holderand H. Keller, "High-temperature superconductors: underlying physics and applications," Zeitschrift für Naturforschung B, vol. 75, pp. 3-14, 2020.

[5] P. Chaudhary, "Polaronic Mechanism of Superconductivity in Cuprates," International Journal of Superconductivity, 2013.

[6] E. Dogotto, "Reviewing some current theories of high-temperature superconductors," Journal of Minerals, Metals \& Materials Society, vol. 49, no. 10, pp. 18-23, 1997.

[7] A. P. Drozdov et al., "Superconductivity at $250 \mathrm{~K}$ in lanthanum hydride under high pressure," Nature, vol. 569, 2019.

[8] High Temperature Superconductors Wires and Cables: Technologies of Energy Conservation, 2019. Retrieved from https://www.electricalindia.in/high-temperaturesuperconductors-wires-and-cables-technologies-ofenergy-conservation/

[9] M. M. Josephine, "Development Trends of Superconducting Cable Technology for Power Transmission Applications" in Proceedings of the World Congress on Engineering and Computer Science, Vol I, 2015.

[10] J. Khachan and S. Bosi, Superconductivity, 2019. [online] http://www.physics.usyd.edu.au/ khachan/PTF/ Superconductivity.pdf.

[11] C. Kittel C Introduction to Solid State Physics. Wiley India Edition, 2010.

[12] J. Østergaard, J. Okholm, K. Lomholt, and O. Tønnesen, "Energy losses of superconducting power transmission cables in the grid," IEEE Transactions on Applied Superconductivity, vol. 11, no.1, pp. 2375-2378, 2001.

[13] P. Schmuser, Superconductivity, 2019. [Online] http://www.desy.de/p̃schmues/Superconductivity.pdf.

[14] Superconductors: A Super Solution, 2019. [Online] from https://www.esdnews.com.au/superconductorssuper-solution/

[15] M. Takato et al., "High-Temperature Superconducting Cable Technology And Development," Sci. Technical Review, vol. 59, 2005.

[16] S. Tanaka, "High-Temperature Superconductivity," Japanese Journal of Applied Physics, vol. 45 no.1, pp. 9011-9024, 2006.

[17] The ITER TOKAMAK. Magnets, 2019. [online] https://www.iter.org/mach/Magnets 
[18] The World Bank. Electric power transmission and distribution losses, $2019 . \quad$ [online] https://data.worldbank.org/indicator/eg.elc.loss.zs

[19] H. Thomas et al., "Superconducting transmission lines Sustainable electric energy transfer with higher public acceptance?," Renewable and sustainable energy reviews, vol. 55, pp. 59-72, 2016.

[20] T. Tohyama T, "Recent Progress in physics of High-Temperature Superconductor,' Japanese Journal of Applied Physics vol. 51, pp. 1-13, 2012.
[21] M. I. Umo, "The Role of Polarons in Cuprates Hi-Tc Superconductivity," Indian Journal of Material Science, 2014.

[22] T. Valla, I. K. Drozdov, and G. D. Gu, "Disappearance of superconductivity due to vanishing coupling in the over doped $\mathrm{Bi}_{2} \mathrm{Sr}_{2} \mathrm{CaCu}_{2} \mathrm{O}_{8+\delta}$," Nature Communications, vol. 11, no. 1, pp. 1-7, 2020.

[23] "Meissner effect," Wikipedia, https://en.wikipedia.org/wiki/Meissner_effect. 